\title{
Systemic Infection by Methicillin-resistant Staphylococcus pseudintermedius in a Bitch
}

\author{
Jôiciglecia Pereira dos Santos', Valesca Ferreira Machado de Souza', Zayan Silva Pereira', \\ lanei de Oliveira Carneiro², Maria Talita Soares Frade ${ }^{3}$ \& Layze Cilmara Alves da Silva Vieira'
}

\begin{abstract}
Background: Staphylococcus pseudintermedius is an opportunistic pathogen, belonging to the genus Staphylococcus. The methicillin-resistant Staphylococci have the mecA-gene, which confers them with the ability of becoming resistant to methicillin and multiple classes of antimicrobials, which makes the treatment of the affections caused by these specimens difficult. This work describes a case of systemic infection and death by methicillin-resistant S. pseudintermedius, in a canine. Case: A crossbred bitch (Canis lupus familiaris), was admitted to the University Veterinary Hospital of the Federal University of Western Bahia (HVU-UFOB). The main complaint reported by the owner was the presence of mammary nodules and constant nasal secretion. During the clinical examination was observed reactivity in the popliteal and left submandibular lymph nodes, pale mucous membranes, stomatitis, bilateral mucopurulent nasal secretion, abdominal pustules, serous secretion in the inguinal mammary gland and focal alopecia on the dorsum. On auscultation, was identified only arrhythmia and the other physiological parameters of the animal were within normality for the species. Samples of the nasal secretion and of the secretion from the abdominal pustules were collected, and sent to the Veterinary Microbiology Laboratory of the same institution. The samples collected were sown in 5\% Blood Agar (BA), Sabouraud Agar (SAB) and MacConkey Agar (MCK), after $24 \mathrm{~h}$ was observed in BA the growth of macroscopically white colonies, with a humid aspect, creamy consistency, with presence of catalase and $\alpha$-hemolysis. Microscopically, was observed the presence of Gram-positive cocci, suggestive of Staphylococcus sp. Microscopically, was observed the presence of Gram-positive cocci, suggestive of Staphylococcus sp. In the MALDI-TOF mass spectrometry, was identified S. pseudintermedius. Enrofloxacin [Enrotrat tab® $25 \mathrm{mg}, 5 \mathrm{mg} / \mathrm{kg}$, SID, PO, 5 days] was prescribed and a follow-up consultation was requested. Two weeks after leaving the University Veterinary Hospital, the animal was admitted in emergency and was submitted to the support protocol and died during the procedure. After the owner's authorization, the anatomopathological examination was carried out, and fragments of the liver, lung and kidney were collected, in addition to sample of the liquid of the abdominal cavity for microbiological examination, and was evidenced the growth of S. pseudintermedius in all the specimens. The bacterium's susceptibility to 19 antibiotics was tested, and a high degree of resistance was found, with sensitivity only to amoxicillin+ clavulanate $(20-10 \mu \mathrm{g})$, chloramphenicol $(30 \mu \mathrm{g})$ and vancomycin $(30 \mu \mathrm{g})$. Given the detection of MRSP in Chromogenic Agar and in cefoxitin disks, all the specimens were MRSP positive.

Discussion: The diagnosis based on the bacteriological culture and anatomopathological findings were essential for the confirmation of the clinical presentation of septicemia. The isolation of S. pseudintermedius in all the analyzed samples, associated to the identification by MALDI-TOF Mass Spectrometry confirmed the clinical suspicion of systemic infection. Based on the result of Antimicrobial susceptibility testing (AST) and phenotypic tests, it was evidenced that all the isolates were MRSP positive, presenting multiple resistance to antibiotics, which may have interfered in the efficiency of the treatment. The results obtained in this report are worrying and signal the need for the implementation of phenotypical researches associated to anti-microbial susceptibility tests in bacteria isolated from animals attended in veterinary clinics and hospitals, in order to monitor and avoid the dissemination of pathogens with a multi-resistant profile.
\end{abstract}

Keywords: antimicrobial resistance, bacteriological examination, anatomopathological examination. 


\section{INTRODUCTION}

The genus Staphylococcus contains more than 40 species of microorganisms which colonize the skin, mucous membranes, gastrointestinal tract, among other areas of the body of humans and animals $[1,5,6,18]$. In this genus, the agents with greater clinical relevance in dogs, are Staphylococcus aureus and Staphylococcus pseudintermedius $[13,14,20]$. These bacteria are commensal colonizers, but can acts as opportunists, causing several affections in humans and animals [7,8,10,12,16-18].

The methicillin-resistant Staphylococcus carry the mecA gene, which confers the ability of becoming resistant to methicillin and to multiple classes of antimicrobials $[5,6,8,16]$. Methicillin-resistant $S$. aureus (MRSA) and methicillin-resistant $S$. pseudintermedius (MRSP) represent a great threat to human and animal health being, therefore, a theme of One Health $[1,6,8,14,18]$. MRSA causes serious infections in humans. The presence of this bacterium has already been reported in production animals, birds, equines, dogs, cats and horses $[6,8,18,19]$. MRSP is detected mainly in dogs with pyoderma, wounds and otitis $[7,18,20]$.

This work reports a case of systemic infection by methicillin-resistant $S$. pseudintermedius in a dog attended at the University Veterinary Hospital of the Federal University of Western Bahia (HVU-UFOB) in the municipality of Barra-BA.

\section{CASE}

A 12-year-old crossbred bitch weighing $6.2 \mathrm{~kg}$, was attended at the HVU-UFOB. During the anamnesis, the animal's owner reported the presence of nodules in the mammary glands and constant nasal secretion, in addition to use of antibiotics without a veterinarian's prescription. In the clinical examination were observed pale mucous membranes, reactivity in the popliteal and left submandibular lymph nodes, stomatitis, light bilateral nasal secretion with a mucopurulent aspect, small abdominal pustules, serous secretion in the inguinal mammary gland and focal alopecia in the dorsum region. Heart rate (76 BPM), respiratory rate (32 Breaths/ min) and body temperature $\left(37.7^{\circ} \mathrm{C}\right)$, within ranges of normality. On cardiopulmonary auscultation, arrhythmia was identified. Samples of the nasal secretion and of the secretion of the abdominal pustules were collected using sterile swabs $\left(\text { Absorve }^{\circledR}\right)^{1}$ stored in Stuart medium (HiMEDIA M306 $\left.{ }^{\circledR}\right)^{2}$ and sent to the Veterinary
Microbiology Laboratory of the same institution. The initial clinical suspicion was pyoderma, therefore an enrofloxacin-based broad-spectrum antibiotic was prescribed [Enrotrat tab ${ }^{\circledR 3} 25 \mathrm{mg}, 5 \mathrm{mg} / \mathrm{kg}$, SID, PO, for 5 consecutive days], and a follow-up consultation of the patient was requested for monitoring at the end of the treatment.

The swabs were sown by the depletion technique in Petri Dishes containing the media 5\% Blood Agar (HiMEDIA M073 $\left.{ }^{\circledR}\right)^{4}$, Sabouraud Agar (HiMEDIA M063 $\left.{ }^{\circledR}\right)^{4}$ and MacConkey Agar (HiMEDIA M081 $\left.{ }^{\circledR}\right)^{4}$. Subsequently incubated at $37^{\circ} \mathrm{C}$ in aerobiosis, and readings were carried out at 24, 48 and $72 \mathrm{~h}$. After $24 \mathrm{~h}$ growth was found in $5 \%$ Blood Agar of macroscopically white colonies, with a humid aspect, creamy consistency, with catalase and presence of $\alpha$-hemolysis. (Figure 1A). Microscopically, was observed the presence of Gram-positive cocci, suggestive of S. pseudintermedius, later confirmed by the Matrix Associated Laser Desorption-Ionization - Time (MALDI-TOF) method [3].

Two weeks after being discharged from the HVU, the animal was admitted in the emergency in a state of hypovolemic shock, being submitted to the stabilization protocols, however without success. After the owner's authorization, the body was sent for anatomopathological examination.

During the necropsy were collected fragments of the liver, lung and kidney, and liquid from the abdominal cavity for microbiological examination, and, in a similar manner to the previously described methodology was evidenced the growth of S. pseudintermedius in all the specimens (Figure 1A).

The antimicrobial susceptibility profile was performed according to the disk diffusion method proposed by the Clinical and Laboratory Standards Institute - CLSI (2009) [9]. The following antibiotics were added in Mueller Hinton Agar (HiMEDIA M173 $\left.{ }^{\circledR}\right)^{4}$ culture medium: Amoxicillin + Clavulanate (20-10 $\mu \mathrm{g})$, Ampicillin $(10 \mu \mathrm{g})$, Cephalexin $(30 \mu \mathrm{g})$, Erythromycin $(15 \mu \mathrm{g})$, Gentamicin $(10 \mu \mathrm{g})$, Oxacillin $(1 \mu \mathrm{g})$, Tetracycline $(30 \mu \mathrm{g})$, Cefalotin $(30 \mu \mathrm{g})$, Cefepime $(30 \mu \mathrm{g})$, Ceftazidime $(30 \mu \mathrm{g})$, Cefotaxime $(30 \mu \mathrm{g})$, Amikacin $(30 \mu \mathrm{g})$, Ceftriaxone $(30 \mu \mathrm{g})$, Azithromycin, $(15 \mu \mathrm{g})$, Cefaclor $(30 \mu \mathrm{g})$, Tobramycin $(10 \mu \mathrm{g})$, Cefoxitin $(30 \mu \mathrm{g})$, Vancomycin $(30 \mu \mathrm{g})$ and Chloramphenicol $(30 \mu \mathrm{g})(\text { Laborclin })^{5}$. The plates were placed in a bacteriological incubator at $35-37^{\circ} \mathrm{C}$ for $24 \mathrm{~h}$. After 


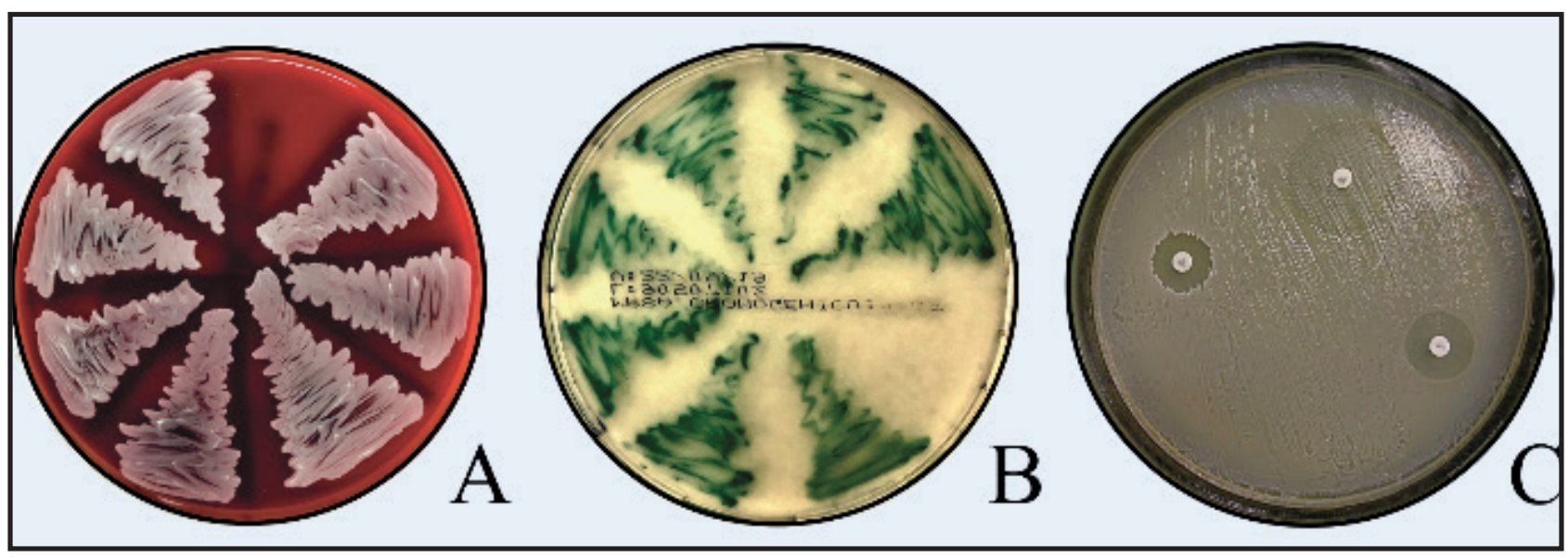

Figure 1. Staphylococcus pseudintermedius. A- White colonies in 5\% blood Agar medium, with a humid aspect, isolated from skin, liquid from the abdominal cavity, fragments of liver, lung and kidney. B- Green colonies in Chromogenic Agar, showing to be MRSP positive. C- Disk Diffusion Method in in Muller Hinton Agar medium, demostrating resistance to Vancomycin and heteroresistance to Cefoxitin and Oxacillin.

the period of incubation the reading of the diameter of the bacterial growth inhibition halo was performed. As quality control, was used the standard strain of the American Type Culture Collection (ATCC): Staphylococcus aureus $\mathrm{n}^{\circ} 25923$. The bacterium demonstrated a high degree of resistance, being sensitive only to Amoxicillin + Clavulanate $(20-10 \mu \mathrm{g})$, Chloramphenicol $(30 \mu \mathrm{g})$ and Vancomycin $(30 \mu \mathrm{g})$.

The detection of MRSP was performed with

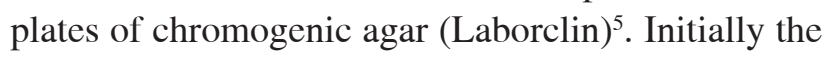
chromogenic agar plates were placed in a bacteriological incubator at $35^{\circ} \mathrm{C}$. Then, with a sterile loop, simple streaking was performed of all the specimens in the surface of the culture medium. Subsequently the chromogenic agar plates were stored for $24 \mathrm{~h}$ at $35^{\circ} \mathrm{C}$. After the incubation period, the reading of the test was carried out according to the recommendations of the manufacturer (Laborclin) ${ }^{5}$ and were visualized colonies bluish-green in color, characteristic of MRSP positive (Figure 1B). For the confirmation of the resistance to methicillin in S. pseudintermedius was performed by the Disk Diffusion Method with Cefoxitin ${ }^{5}$, Oxacillin ${ }^{5}$ and Vancomycin ${ }^{5}$ (Figure 1C), and was evidenced resistance to Vancomycin and heteroresistance to Cefoxitin and Oxacillin. In the diffusion test, the bacterial inoculum was sown on the surface of a Petri dish containing the Mueller-Hinton Agar (MHA) and then, were placed on the surface of the medium, Cefoxitin, Oxacillin and Vancomycin disks. The cultures were incubated at $37^{\circ} \mathrm{C}$ for $24 \mathrm{~h}$. After this period, the reading of the dishes was carried out for the observation of the result.

In the necropsy were observed slightly icteric ocular, oral and vulvar mucous membranes. In the ventral abdominal region, there was the presence of multiple ulcers, which extended to the medial surface of the pelvic limbs, varying from 0.5 to $1 \mathrm{~cm}$ of extension, with a depressed center varying from whitish to red and elevated and irregular edges, at times draining purulent content (Figure 2A). The superficial inguinal lymph nodes were enlarged. In the abdominal cavity, there was the presence of serosanguinolent liquid (approximately $67 \mathrm{~mL}$, petechiae in the omentum, close to the cranial border of the spleen. The spleen was also increased in volume, the cut surface revealed protrusion of the white pulp (Figure 2B). Liver with bulging borders and accentuation of the lobular pattern. The kidneys were pallid and reduced in volume, with reddish irregular areas in the subcapsular surface. Intestines with liquefied and greasy content (milky-yellow). Stomach with reddish content and multiple erosions and ulcers mainly in the pyloric antrum region. The lungs were not collapsed and diffusely reddish. In the pericardial sac there was a discreet quantity of translucent liquid (hydropericardium) and in the endocardium was observed nodular thickening of the left atrioventricular valve, suggestive of endocardiosis. The uterus presented nodular cystic hyperplasia and presence of fibrinosupurative content. Were collected fragments of organs of the abdominal cavity, thoracic cavity and central nervous system, these were fixed in $10 \%$ formaldehyde, and processed by the routine technique for histopathological examination, stained using hematoxylin and eosin. Microscopically, the significant findings were found in the skin, lymph node, spleen, heart and encephalon. In the skin, there was superficial and deep dermatitis, mixed, with the 
J.P. Santos, V.F.M. Souza, Z.S. Pereira, et al. 2021. Systemic Infection by Methicillin-resistant Staphylococcus pseudintermedius

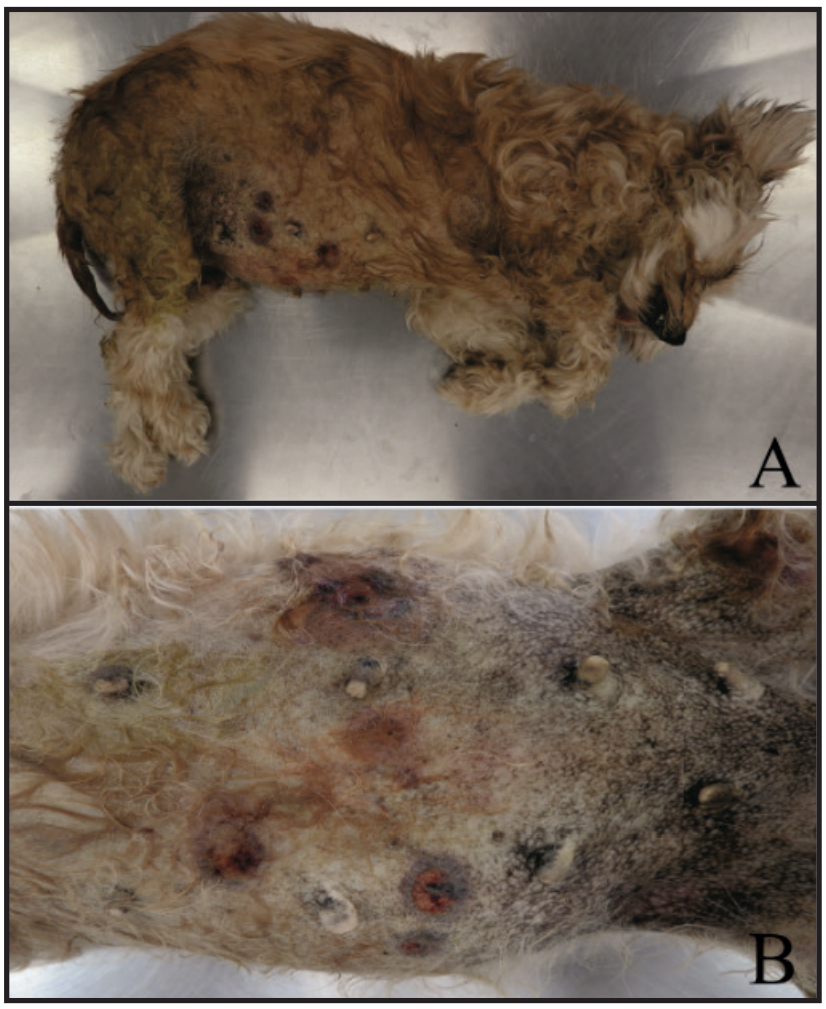

Figure 2. A 12-year-old crossbred bitch. A- Left lateral decubitus, evidence of abdominal bulging. B- Skin of the ventral region with irregular ulcerated multifocal areas with depressed center varying from whitish to red and irregular and elevated borders, draining purulent content.

presence of lymphocytes, plasmocytes, macrophages, neutrophils and mast cells, multifocal to coalescing, associated to the ulceration of the epidermis. There was also the presence of inflammatory cells and fibrin filaments in the lumen of the vessels. In the lymph node there was lymphoid rarefaction and in the white pulp of the spleen was observed infiltrates of plasmocytes and macrophages (plasmocytosis and histiocytosis of the white pulp). In the subepicardial layer of the heart there was the discreet presence of inflammatory infiltrate of lymphocites, plasmocytes and macrophages. In the ventricular lumen was observed a great quantity of inflammatory cells interspersed by fibrin filaments. In the brain, was observed leukocyte esterase in the vessels of the leptomeninges. Based on the macro and microscopic findings, was established the anatomopathological diagnosis of dermatitis/panniculitis associated to septicemia.

\section{DISCUSSION}

Staphylococcus pseudintermedius is a component of the normal skin microbiota of dogs and can be involved in conditions such as pyoderma, endocarditis, omphalophlebitis, pododermatitis, toxemia and septicemia [10,12,16-18]. The environmental contamination and the contact with other animals and humans carriers of MRSP may have been an acquisition route of the bacterium, corroborating with previous studies $[11,13,17]$. Also, the indiscriminate use of antibiotics without the monitoring of the resistance profile, added to events of immunosuppression associated to the animal, such as age, poor nutrition, infections by parasitic, bacterial or viral agents, not investigated in this work, may have favored the development of resistance mechanisms by the pathogen, culminating with the incorporation of $m e c A$ genes responsible for the resistance to methicillin $[8,13]$.

The clinical history added to the necropsy findings, to the results of the microbiological diagnosis of isolation and mass spectrometry, confirmed the presence of the agent $S$. pseudintermedius in the tissues, being therefore the agent responsible for the sepsis in the animal studied. Systemic infections in dogs caused by the isolated agent have a low frequency of mortality [16]. However, this pathogen can be associated with a high morbidity rate especially in immunocompromised dogs. This distribution gives the dimension of the problem and demonstrates the importance of the application of the sensitivity test in the clinical routine for the treatment of bacterial diseases.

Methicillin-resistant staphylococci are also resistant to multiple classes of drugs [19,20], complicating the therapeutics and clinical improvement of the infection. The pathogen isolated presented resistance to $84.21 \%(16 / 19)$ of the drugs tested, and was sensitive only to Amoxicillin + Clavulanate, Chloramphenicol and Vancomycin. Similar results were found by Silva [10], who obtained a good sensitivity of the association of amoxicillin + clavulanic acid in strains of Staphylococcus spp. from cutaneous lesions of dogs with superficial pyoderma, which can be attributed to the fact that this acid is an inhibitor of the $\beta$-lactamases produced by the staphylococci. In the work described by Wan [19] about methicillin-resistant S. pseudintermedius causing recurrent pyoderma in a dog, was observed that amongst other antibiotics the chloramphenicol proved to be efficient against the MRSP, this corroborates with the results found in this report. In Human Medicine, the Vancomycin is a drug frequently used in the treatment of infections caused by multiresistant staphylococci [10]. The sensitivity identified in the bacterium of the present work to this antimicrobial, may be justified by 
the its restricted use in companion animals. In Veterinary Medicine, the use of vancomycin is recommended when the susceptibility tests have showed resistance to all the other available antimicrobials [10]. The antibiogram of this work, due to the lack of financial support at the moment of the clinical consultation, was only carried out after the death of the animal, using the frozen bacterial strains. This invalidated the access of the veterinary doctor to more efficient therapeutic options during the clinical treatment, a fact that may have influenced the outcome of this case.

The dermatitis and panniculitis were associated to the agent $S$. pseudintermedius. The deposition of inflammatory cells and deposition of fibrin in the lumen of the vessels, indicate septicemia, which was evidenced by the isolation of the agent in the tissues and liquids collected for microbiological examination.

The death of the animal probably occurred due to septic shock, although it is not very common cases of septicemia and death by $S$. pseudintermedius. The physiopathology of the septic shock is quite complex involving several mechanisms and intrinsic factors of the patient, as well as of the pathogen involved in the clinical picture $[2,4,15]$. The organic system probably suffered a dysfunction due to the action of endotoxins produced by the pathogen, which culminated with the death of the animal.

This work described a report of systemic infection and death by methicillin-resistant Staphylococcus pseudintermedius in a bitch. It is important to emphasize that the etiological agent involved is opportunistic and can cause disorders in the One Health sphere. The diagnosis based on the bacteriological culture, MALDI- TOF and in the anatomopathological findings were essential for the confirmation of the agent and clinical presentation, enabling the study of the dimension of the affection. Based on the result of the antibiogram, the resistance of the isolate to methicillin and to multiple antimicrobial classes was proven. Therefore, to identify the susceptibility profile of the microorganisms to therapeutic agents, it is necessary to adopt microbiological techniques, such as the antibiogram, which is a guiding tool at the time of choosing the treatment to be employed. Failures may lead to systemic infections, which are potentially fatal when not diagnosed early on. The correct diagnosis is fundamental for the adoption of the effective therapy and reduction of the high morbidity and mortality rates from septic shock.

\section{MANUFACTURERS}

${ }^{1}$ Cral artigos para laboratório Ltda. Cotia, SP, Brazil.

${ }^{2}$ HiMedia Laboratories Unip. Ltd. Mumbai, India.

${ }^{3}$ Ourofino Saúde Animal Ltda. Cravinhos, SP, Brazil.

${ }^{4}$ Dsyslab - Produtos e Equipamentos para Laboratórios, Clínicas e

Hospitais. Curitiba, PR, Brazil.

${ }^{5}$ Laborclin Produtos para Laboratórios Ltda. Pinhais, PR, Brazil.

Declaration of interest. The authors report no conflicts of interest. The authors alone are responsible for the content and writing of the paper.

\section{REFERENCES}

1 Arias M.V.B \& Carrilho C.M.D.M. 2012. Resistência antimicrobiana nos animais e no ser humano. Há motivo para preocupação? Semina: Ciências Agrárias. 33(2): 775-790.

2 Barbosa C.B., Alves F.S., Beier L.S., Faleiros R.R. \& Freitas P.M.C. 2016. Fisiopatologia e terapia do cão com sepse: revisão. PUBVET. 10(1): 13-20.

3 Barcelos M.M., Martins L., Grenfell R.C., Juliano L., Anderson K. L., Santos M.V \& Gonçalves J.L. 2019. Comparison of standard and on-plate extraction protocols for identification of mastitis-causing bacteria by MALDI-TOF MS. Brazilian Journal of Microbiology. 50: 849-857.

4 Basso P.C., Müller D.C.M. \& Serafini G.M.C. 2012. Pathophysiology and management of sepsis and systemic inflammatory response syndrome -literature review Medvep - Revista Científica de Medicina Veterinária - Pequenos Animais e Animais de Estimação. 10(34): 430-436.

5 Botoni L.S., Scherer C.B., Silva R.O., Coura F.M., Heinemann M.B., Paes Leme F.O. \& Costa Val A.P. 2016. Prevalência e suscetibilidade in vitro de Staphylococcus pseudintermedius resistente à meticilina (MRSP) oriundos de pele e narinas de cães com piodermite superficial. Pesquisa Veterinária Brasileira. 36(12): 1178-1180.

6 Bourguignon E., Viçosa G.N., Corsini C.M.M., Moreira M.A.S., Nero L.A. \& Conceição L.G. 2016. Description of Methicillin-resistant Staphylococcus pseudintermedius from canine pyoderma in Minas Gerais state, Brazil. Arquivo Brasileiro de Medicina Veterinária e Zootecnia. 68(2): 299-306. 
7 Cerqueira E.S \& Almeida R.C.C. 2013. Methicillin-resistant Staphylococcus aureus (MRSA) in foods of animal origin: A systematic review. Revista do Instituto Adolfo Lutz. 72(4): 268-281.

8 Cuny C., Wieler L.H. \& Witte W. 2015. Livestock-Associated MRSA: The Impact on Humans. Antibiotics. 4(4): 521-543.

9 Clinical Laboratory Standards Institute. 2009. Performance Standards for Antimicrobial Disk Susceptibility Tests. 10th edn. CLSI supplement M2 A10, PA: Clinical Laboratory Standards Institute, 76p.

10 Da Silva A.P., Schmidt C., Vargas A.C., Maboni G., Rampelotto C., Schwab M.L., Escobar T.P. \& Amaral A.S. 2014. Antimicrobial susceptibility of Staphylococcus spp. isolated from canine superficial pyoderma. Pesquisa Veterinária Brasileira. 34(4): 355-361.

11 Duijkeren E.V., Kamphuis M., VanderMije I.C., Laarhoven L.M., Duim B., Wagenaar J.A. \& Houwers D.J. 2011. Transmission of methicillin-resistant Staphylococcus pseudintermedius between infected dogs and cats and contact pets, humans and the environment in households and veterinary clinics. Veterinary Microbiology. 150(3-4): 338-343.

12 Grönthal T., Eklund M., Thomson K., Piiparinen H., Sironen T. \& Rantala M. 2017. Antimicrobial resistance in Staphylococcus pseudintermedius and the molecular epidemiology of methicillin-resistant S. pseudintermedius in small animals in Finland. Journal of Antimicrobial Chemotherapy. 72(4): 1021-1030.

13 Harrison E.M., Weinert L.A., Holden M.T.G., Welch J.J., Wilson K., Morgan F.J.E., Harris S.R., Loeffler A., Boag A.K., Peacock S.J., Paterson G.K., Waller A.S., Parkhill J. \& Holmes M A. 2014. A Shared Population of Epidemic Methicillin-Resistant Staphylococcus aureus 15 Circulates in Humans and Companion Animals. American Society for Microbiology. 5(3): DOI: 10.1128/mBio.00985-13

14 Ishihara K., Shimokubo N., Sakagami A., Ueno H., Muramatsu Y., Kadosawa T., Yanagisawa C., Hanaki H., Nakajima C., Suzuki Y. \& Tamura Y. 2010. Occurrence and molecular characteristics of methicillin-resistant Staphylococcus aureus and methicillin-resistant Staphylococcus pseudintermedius in an Academic Veterinary Hospital. Applied and environmental microbiology. 76: 5165-5174.

15 Mcgavin M.D. \& Zachary J.F. 2013. Bases da Patologia em Veterinária. 5.ed. Rio de Janeiro: Elsevier, 196p.

16 Pipan M.Z., Švara T., Zdovc I., Papi B., Avberšek J., Kušar D. \& Mrkun J. 2019. Staphylococcus pseudintermedius septicemia in puppies after elective cesarean section: confirmed transmission via dam's milk. BMC Veterinary Research. 15: 41.

17 Stegmann R., Burnens A., Maranta C.A \& Perreten V. 2010. Human infection associated with methicillin-resistant Staphylococcus pseudintermedius ST71. Journal of Antimicrobial Chemotherapy. 65(9): 2047-2048.

18 Ventura F.V.C \& Oliveira S.T. 2011. Etiologia e terapia das endocardites bacterianas em cães - revisão. Arquivos de Ciências Veterinárias e Zoologia da UNIPAR. 14(2): 145-150.

19 Wan J. 2014. A case of methicillin-resistant Staphylococcus pseudintermedius (MRSP) pyoderma in a Labrador retriever dog. Journal of Internal Veterinary Medicine. 55(11): 1100-1101.

20 Weese J.S. \& van Duijkeren E. 2010. Methicillin resistant Staphylococcus aureus and Staphylococcus pseudintermedius in veterinary medicine. Veterinary Microbiology. 140(3): 418-429. 